\title{
Dark Matter Indirect Detection
}

\author{
Stefano Profumo ${ }^{1, a}$ \\ ${ }^{1}$ University of California, Santa Cruz, and Santa Cruz Institute for Particle Physics, \\ 1156 High St., Santa Cruz CA 95064, USA
}

\begin{abstract}
This is an overview of recent developments and future prospects in the search for dark matter and new physics with astronomical and astro-particle techniques.
\end{abstract}

Indirect detection refers to the exercise of seeking the hopefully unique astronomical, astro-particle, or cosmological signals that probe the particle nature of the Dark Matter (DM). The energy scale of such signals depends on the DM mass. On largely model-independent grounds, the DM mass is constrained from below by quantum effects having to exist on scales smaller than the size of the smallest gravitationally collapsed objects, and from above from the requirement that macroscopic DM particles not disrupt the stability of long-lived structures in the universe, such as globular clusters and galactic disks. As a result, the landscape of possible DM masses ranges over more than 90 orders of magnitude, from approximately $10^{-22} \mathrm{eV}$ up to $10^{70} \mathrm{eV}$.

At the lowest mass scale, "fuzzy" or "wave" dark matter has spawned numerous exciting new ideas both in experiments and in observations. While strong constraints exist from observations of the $21 \mathrm{~cm}$ line, the experimental landscape now includes a number of novel setups inculding ABRACADABRA, LC resonators, HAYSTAC, MW cavities among others.

Observations of an unidentified X-ray line at $3.5 \mathrm{keV}$ in the spectrum of individual and stacked clusters of galaxies as well as in the Galactic center has spurred much interest, as it could arise from the decay of an approximately 7 $\mathrm{keV}$ sterile neutrino to an active neutrino and a photon. Sterile neutrinos are viable dark matter candidates possibly connected with ordinary neutrinos as well as with baryogenesis via leptogenesis. While the $3.5 \mathrm{keV}$ might arise from a previously unaccounted for de-excitation line of K XVIII (a He-like potassium ion), observations of Draco with XMM strongly disfavors a sterile neutrino decay origin. New astrophysical solutions, such as chargeexchange processes, and new theoretical ideas, including "fluorescent" dark matter and axion-like particle conversions have been proposed. A replacement to the Hitomi satellite promises to clarify the observational situation with significantly improved energy resolution.
A new frontier for indirect detection lies in the $\mathrm{MeV}$ range, with a few proposed new instruments that would revolutionize existing observations, such as eASTROGAM and ComPair. An interesting theoretical question lies with what the annihilation of MeV DM particles would look like, and how to evade CMB constraints. Photon production would arise from radiative processes off of charged particles, as well as from the decay of hadrons. A new code, Hazma, allows for the matching of UV complete theories onto hadrons via chiral perturbation theory. Constraints from cosmology appear to severely limit the possible ranges for the scattering cross section of $\mathrm{MeV}$ and sub-MeV DM off of electrons, with dire implications for proposed new experiments.

The Galactic center gamma-ray excess persists, albeit it continues to rely on arguably primitive models for the diffuse gamma radiation in the Galaxy. It was shown that positing that a fraction of the cosmic-ray source are placed in regions of star formation makes the excess largely disappear. It is intriguing that similar excesses exist in the Andromeda galaxy (M31), although cosmic rays are a viable counterpart for the production of gamma rays in that system. An additional excess also exists in the globular cluster Omega Centauri, although again other sources, such as millisecond pulsars, are perfectly reasonable counterparts.

Overall, the prospects of establishing a conclusive DM signal from gamma-ray observations remain weak, although a conclusive detection, for instance of a gamma-ray line at $\mathrm{MeV}$ or $\mathrm{TeV}$ energies, remains a distinct possibility.

A further puzzling signal is a rising positron fraction, conclusively measured by HEAT, Pamela, Fermi and most recently by AMS-02. Nearby, mature pulsars appear to be likely counterparts, although a recent observational result from the HAWC telescope has brought this possibility into question: specifically, the observation of $\mathrm{TeV}$ halos from inverse Compton scattering of CMB photons in the nebulae surrounding two nearby pulsars, Monogem and Geminga, often invoked as the source of the anomalous positrons, has shown that diffusion within PWNs is highly

ae-mail: profumo@ucsc.edu 
inefficient. If this is the case, high-energy positrons could not travel from those pulsars to Earth, if diffusion is inefficient throughout. However, the likely culprit is inhomogeneous diffusion, a possibility that is currently being tested.

While no excess seems to be present in the spectrum of antiprotons, AMS-02 has reported the tentative detection of massive antinuclei such as anti-Helium-3 and antiHelium-4. If this is indeed confirmed, DM annihilation might be invoked as a solution, albeit at the expense of stretching the coalescence picture that leads to the prediction of the formation of heavy antinuclei from DMproduced antinucleons.

The detection of gravitational waves has brought to the forefront primordial black holes (PBH) as possible DM candidates. LIGO BH's can actually be of primordial origin, as perhaps suggested by the low measured spins for most of the recently reported events (although superradiance could also be responsible for the low observed spin). CMB limits are currently being re-evaluated, but a conclusive evidence for a primordial origin might lie with the detection of a sub-Chandrasekhar mass merging $\mathrm{BH}$.
It was recently realized that finite-size source effects can play a critical role in alleviating microlensing constraints deriving from the observation of stars in the M31 Galaxy with the SUBARU HSC camera. This observation has effectively re-opened the landscape of asteroid-mass $\mathrm{PBHs}$ as possible contributors to $100 \%$ of the DM in the universe.

PBH with much lighter masses, around $1,000 \mathrm{~kg}$, could have evaporated to both dark matter and right-handed neutrinos, a possibility dubbed melanopogenesis. Much lighter objects, around the Planck scale, might be stable because of quantum gravity or extremality, and be stuck with a relic electric charge. If this is the case both large neutrino detectors, and so-called paleo-detectors could soon probe this possible class of ultra-heavy particle DM candidates.

On the horizon, the combination of new observational facilities such as the Cherenkov Telescope Array, new $\mathrm{MeV}$ telescopes, and new radio surveys, new ideas on direct detection of light dark matter candidates, the fruitful interaction of theorists and observers, and the readiness of experimentalists alike promises to usher an era of discovery. 